\title{
PW03-014 - TLR4 and MEFV variants are Behçet's risk factors
}

\author{
Y Kirino ${ }^{1,2^{*}}$, Q Zhou $^{2}$, Y Ishigatsubo ${ }^{1}$, N Mizuki ${ }^{3}$, Y Kim ${ }^{4}$, DL Kastner ${ }^{2}$, A Gül ${ }^{5}$, EF Remmers ${ }^{2}$ \\ From 7th Congress of International Society of Systemic Auto-Inflammatory Diseases (ISSAID) \\ Lausanne, Switerland. 22-26 May 2013
}

\section{Introduction}

Genome-wide association studies (GWAS) are a powerful means for identifying genes with disease-associated common variants, but they are not well-suited to detect genes with disease-associated rare or low-frequency variants. It has long been debated whether the innate immune system is involved in the pathogenesis of Behçet's disease (BD) but genetic evidence to support this hypothesis is sparse.

\section{Objectives}

To determine whether rare and low frequency variants in genes involved in innate immunity are associated with BD.

\section{Methods}

In the current study, non-synonymous variants (NSVs) identified by deep exonic resequencing of 10 genes found by GWAS (IL10, IL23R, CCR1, STAT4, KLRK1, KLRC1, KLRC2, KLRC3, KLRC4, and ERAP1) and 11 genes selected for their role in innate immunity (IL1B, IL1R1, IL1RN, NLRP3, MEFV, TNFRSF1A, PSTPIP1, CASP1, PYCARD, NOD2, and TLR4) were evaluated for $\mathrm{BD}$ association in Japanese and Turkish populations. A differential distribution of the rare and low frequency NSVs of each gene in 2461 BD cases compared with 2458 controls was evaluated by three different burden tests.

\section{Results}

By stringent criteria requiring at least one burden test with study-wide significance $(\mathrm{p}<0.0024)$ and a corroborating test with at least nominal significance $(\mathrm{p}<0.05)$, rare and low frequency NSVs in one GWAS-identified gene, IL23R $\left(\mathrm{p}=6.9 \times 10^{-5}\right)$, and one gene involved in innate immunity, TLR4 $\left(\mathrm{p}=8.0 \times 10^{-4}\right)$, were associated with BD. In addition, damaging or rare damaging NOD2 variants were nominally significant across all three burden tests applied ( $\mathrm{p}=0.0063$ to 0.045 ). Furthermore, carriage of $M E F V-\mathrm{M} 694 \mathrm{~V}$, but not other $M E F V$ mutations known to cause recessively inherited familial Mediterranean fever, conferred BD risk in the Turkish population $\left(\mathrm{OR}=2.65, \mathrm{p}=1.8 \times 10^{-12}\right)$.

\section{Conclusion}

Rare and low frequency NSVs of two novel BD-associated genes, $M E F V$ and TLR4, implicate innate immune and bacterial sensing mechanisms in BD pathogenesis. Furthermore, disease-associated IL23R rare and low frequency NSVs add to the common variant GWAS evidence implicating this locus.

\section{Disclosure of interest}

None declared.

\section{Authors' details}

'Department of Internal Medicine and Clinical Immunology, Yokohama City University Graduate School of Medicine, Yokohama, Japan. ${ }^{2}$ Inflammatory Disease Section, Medical Genetics Branch, NIH/NHGRI, Bethesda, USA. ${ }^{3}$ Department of Ophthalmology and Visual Science, Yokohama City University Graduate School of Medicine, Yokohama, Japan. ${ }^{4}$ Genometrics Section, Inherited Disease Research Branch, NIH/NHGRI, Baltimore, USA. ${ }^{5}$ Istanbul Faculty of Medicine, Department of Internal Medicine, Division of Rheumatology, Istanbul University, Istanbul, Turkey.

Published: 8 November 2013

doi:10.1186/1546-0096-11-S1-A240

Cite this article as: Kirino et al:: PW03-014 - TLR4 and MEFV variants are Behçet's risk factors. Pediatric Rheumatology 2013 11(Suppl 1):A240. 